\title{
PERSPECTIVITY IN PROJECTION LATTICES ${ }^{1}$
}

\section{PETER A. FILLMORE}

Let $A$ be a $w^{*}$-algebra and $L$ its projection lattice. It is well known [3] that projections perspective in $L$ are linked by a partial isometry in $A$, and that these two notions coincide precisely when the algebra $A$ is finite. We show in this paper that, in any $w^{*}$-algebra, perspectivity enjoys virtually every property, other than additivity, of the relation $\sim$ of partial isometry equivalence. These properties are established by means of

Theorem 1. Projections $p, q \in L$ are perspective in $L$ if and only if they are unitarily equivalent in $A$.

In one direction this is immediate, for if $r \in L$ is a common complement for $p$ and $q$, then $r^{\prime}=1-r$ is a common complement for $p^{\prime}$ and $q^{\prime}, p \sim q, p^{\prime} \sim q^{\prime}$, and $p$ and $q$ are unitarily equivalent.

We begin the converse with two lemmas (valid in any orthomodular lattice $L$ ) concerning the additivity of perspectivity. Recall that an orthomodular lattice is a lattice with an orthocomplementation $a \rightarrow a^{\prime}$ such that $b=a+a^{\prime} \cap b$ whenever $a \leqq b$. (The symbol + will be used for the lattice join if the summands are orthogonal.) It is easily seen that this condition is equivalent to the following:

$$
a \leqq c \text { implies }(a+b) \cap c=a+b \cap c .
$$

Lemma 1. If $a, b, c, d \in L$ and $a \cup c \perp b \cup d$, then $(a+b) \cap(c+d)$ $=a \cap c+b \cap d$.

To prove this one can imitate the proof in [5, Theorem 1.2], observing that our assumption permits replacement of the modular law by the restricted version of it given above.

Lemma 2. If $a, b, c, d, e, f \in L, a$ and $b$ are perspective in $[0, e], c$ and $d$ are perspective in $[0, f]$, and $e \perp f$, then $a+c$ and $b+d$ are perspective in $[0, e+f]$, as are $a+f$ and $b+f$.

Proof. Let $x$ be a common complement for $a$ and $b$ in $[0, e], y$ for $c$ and $d$ in $[0, f]$, and put $z=x+y$. Plainly, $a \cup c \cup z=e+f=b \cup d \cup z$, and, since $a \cup x \perp c \cup y$, we have

$$
(a \cup c) \cap z=(a+c) \cap(x+y)=a \cap x+c \cap y=0
$$

Received by the editors February 7,1964.

1 This work was supported by a grant from the National Science Foundation. 
by Lemma 1. Similarly $(b \cup d) \cap z=0$, and the second part of the lemma follows in an analogous fashion.

For the remainder of the paper $L$ will denote the projection lattice of a $w^{*}$-algebra on a Hilbert space $H$. We are indebted to Professors Halperin and Kaplansky for simplifications of several of the subsequent proofs.

Lemma 3. If $p, q \in L, p \cap q=0$, and $p \sim q$, then $p$ and $q$ are perspective in $[0, p \cup q]$.

Proof. Following [ $2 ;$ p. 9], if $u \in A$ implements the equivalence of $p$ and $q$ and $\lambda$ is a complex number with $|\lambda|<1$, then the range projection of $(1+\lambda u) p$ has the required properties.

Lemma 4. If $p, q, r \in L$ satisfy $q \sim p \leqq q$ and $p \lesssim r \leqq q^{\prime}$, then $p$ and $q$ are perspective in $[0, q+r]$.

Proof. $p \sim s \leqq r$, and let $u, v$ be partial isometries in $A$ with $u^{*} u=q$, $u u^{*}=s, v^{*} v=q$, and $v v^{*}=p$. For $n \geqq 1$ denote by $c_{n}$ the range projection of $\left(v^{n}+(1 / n)\left(1+u v^{n}\right)\right)(q-p)$, and let $c=\bigcup c_{n}$. Of course $c \in L$, and it will be shown that $c$ is a common complement for $p$ and $q$ in $[0, p \cup c]$ by showing (1) $q \leqq p \cup c$ and (2) $q \cap c=0$.

Let $e \in L, e \perp p \cup c$. Then, for each $n \geqq 1, e\left(v^{n}+(1 / n)\left(1+u v^{n}\right)\right)(q-p)$ $=0$, whence $e\left(1+u v^{n}\right)(q-p)=0$ and so $e(q-p)=-(1 / n)$ $\sum_{k=1}^{n} e u v^{k}(q-p)$. For each $\alpha \in H$ we have

$$
\|e(q-p) \alpha\|=\frac{1}{n}\left\|\sum_{k=1}^{n} e u v^{k}(q-p) \alpha\right\| \leqq \frac{1}{n} \sqrt{ } n\|(q-p) \alpha\|,
$$

so that $e(q-p)=0, e \perp q$, and $q \leqq p \cup c$.

To prove (2) suppose that $\alpha$ is an element in the range of $q \cap c$. For any $\epsilon>0$ there are elements $\alpha_{k}$ in the range of $q-p$ such that

$$
\left\|\alpha-\sum\left(v^{k}+\frac{1}{k}\left(1+u v^{k}\right)\right) \alpha_{k}\right\|<\epsilon .
$$

Hence, since $q^{\prime} \alpha=0$, we have

$$
\left\|\sum \frac{1}{k} u v^{k} \alpha_{k}\right\|=\left(\sum \frac{1}{k^{2}}\left\|\alpha_{k}\right\|^{2}\right)^{1 / 2}<\epsilon .
$$

Denoting by $p_{k}$ the range projection of $v^{k}(q-p),(*)$ implies $\left\|p_{k} \alpha-v^{k} \alpha_{k}\right\|$ $<\epsilon$, and, therefore,

$$
\left\|p_{k} \alpha\right\| \leqq \epsilon+\left\|v^{k} \alpha\right\| \leqq \epsilon+k \epsilon .
$$

Since $\epsilon$ is arbitrary, $p_{k} \alpha=0$. Again $\left(\sum p_{k}\right) \alpha=p \alpha$, for, by $(*)$, 
$\left\|p \alpha-\sum v^{k} \alpha_{k}\right\|<\epsilon$ and $\left\|\left(\sum p_{k}\right) \alpha-\sum v^{k} \alpha_{k}\right\|<\epsilon$. Thus $p \alpha=0$, whence $\left\|\sum v^{k} \alpha_{k}\right\|=\left(\sum\left\|\alpha_{k}\right\|^{2}\right)^{1 / 2}<\epsilon$. Finally, $\left\|(q-p) \alpha-\sum(1 / k) \alpha_{k}\right\|<\epsilon$ by $(*)$, so

$$
\|(q-p) \alpha\| \leqq \epsilon+\left\|\sum \frac{1}{k} \alpha_{k}\right\| \leqq \epsilon+\left[\left(\sum\left\|\alpha_{k}\right\|^{2}\right)\left(\sum \frac{1}{k^{2}}\right)\right]^{1 / 2} \leqq K \epsilon,
$$

$K$ fixed. Therefore, $q \alpha=0, \alpha=0$, and $q \cap c=0$.

It is readily verified that $p \cup c \leqq q+r$, and the proof is completed by enlarging $c$ to a common complement for $p$ and $q$ in $[0, q+r]$.

Lemma 5. If $a, b, c \in L$ satisfy $a+b+c \sim a+b$, then there exist $c_{1}, c_{2} \in L$ such that $c=c_{1}+c_{2}, a+c_{1} \sim a$, and $b+c_{2} \sim b$.

Proof. Split the algebra into two summands on which, respectively, $a \lesssim b$ and $b \lesssim a$. On the first summand we can assume that $b$ is purely infinite, for $b$ finite implies $a$ finite, $a+b$ finite, and $c=0$. Hence $b=b_{1}+b_{2}$ with $b \sim b_{1} \sim b_{2}$, so

$$
b+c \leqq a+b+c \sim a+b \lesssim b_{1}+b_{2}=b
$$

and we have $b+c \sim b$. On the summand with $b \precsim a$ we have, similarly, $a+c \sim a$.

LEммA 6. If $a, b, x \in L$ satisfy $a \leqq b$ and $a \lesssim x \lesssim b$, then there exists $y \in L$ such that $y \sim x$ and $a \leqq y \leqq b$.

Proof. Let $x=c+u$ with $a \sim c$, so that $c+u \precsim a+(b-a)$. Split the algebra into two summands on which, respectively, $u \lesssim b-a$ and $b-a \lesssim u$. On the first we have $u \sim w \leqq b-a$, so the element $y=a+w$ meets our requirements. If $b-a \lesssim u$ we have $b=a+(b-a) \precsim c+u$ $=x \lesssim b$, so $b \sim x$ and $y=b$ serves.

Proof of Theorem 1. Let $p, q \in L$ be unitarily equivalent. Drop first to a direct summand in which $p \cap q \lesssim p^{\prime} \cap q^{\prime}$, and split this into two further summands in which $p-p \cap q \lesssim q-p \cap q$ and $q-p \cap q$ $\lesssim p-p \cap q$, respectively. In the first of these we wish to locate $s \leqq p^{\prime} \cap q$ such that $p-p \cap q \sim q-p \cap q-s$. To this end we note that the elements $a=p-p \cap q-p \cap q^{\prime}$ and $b=q-p \cap q-p^{\prime} \cap q$ satisfy $a \cap b^{\prime}=a^{\prime} \cap b=0$. Hence $b^{\prime}$ is a common complement for $a$ and $b$, $a \sim b$, and

$$
q-p \cap q-p^{\prime} \cap q \lesssim p-p \cap q \lesssim q-p \cap q .
$$

Applying Lemma 6 there exists $r \sim p-p \cap q$ such that

$$
q-p \cap q-p^{\prime} \cap q \leqq r \leqq q-p \cap q
$$


and letting $r+s=q-p \cap q$ we have $s \leqq p^{\prime} \cap q$ and $p-p \cap q \sim q-p$ $\cap q-s$ as desired. Now

$$
r+p \cap q \sim(p-p \cap q)+p \cap q \sim q=r+p \cap q+s
$$

so, by Lemma 5 , we have $s=s_{1}+s_{2}$ with $r+s_{1} \sim r$ and $p \cap q+s_{2}$ $\sim p \cap q$. Hence $p-p \cap q \sim r+s_{1}$, and, since these are disjoint, they are perspective in their union $e$ by Lemma 3. Moreover, since $p \cap q$ $\lesssim p^{\prime} \cap q^{\prime}$, Lemma 4 implies that $p \cap q$ and $p \cap q+s_{2}$ are perspective in $f=p \cap q+s_{2}+p^{\prime} \cap q^{\prime}$. Our choice of $s$ is such that $e$ and $f$ are orthogonal, and thus Lemma 2 permits addition of the above perspectivities, thereby giving the perspectivity of $p$ and $q$.

We proceed similarly in the summand in which $q-p \cap q \lesssim p-p \cap q$. In the summand in which $p^{\prime} \cap q^{\prime} \lesssim p \cap q$ we use the fact that $p^{\prime}$ and $q^{\prime}$ are unitarily equivalent to establish as above their perspectivity. But this immediately implies that of $p$ and $q$, and a final addition of perspectivities via Lemma 2 completes the proof.

Corollary. Perspectivity is transitive in the projection lattice of a $w^{*}$-algebra.

Theorem 2 (Cantor-Schroeder-Bernstein). Let $L$ be the projection lattice of $a w^{*}$-algebra. If $p, q \in L$ are each perspective to a subprojection of the other, then they are perspective.

Proof. By Theorem 1 we need only prove the statement with "perspective" replaced by "unitarily equivalent." We have $p \precsim q$ and $q \lesssim p$, and so $p \sim q$. But also $p^{\prime}$ and $q^{\prime}$ are each unitarily equivalent to a subprojection of the other, so that $p^{\prime} \sim q^{\prime}$. Therefore, $p$ and $q$ are unitarily equivalent.

Our next result is an immediate consequence of Topping's results on weakly closed Jordan algebras of self-adjoint operators [6]. However, in this context, a simpler direct proof is available.

Theorem 3 (Generalized COMPARABILITy). Let $L$ be the projection lattice of a $w^{*}$-algebra $A$. For any $p, q \in L$ there is a central projection $e$ of $A$ such that ep is perspective to a subprojection of eq and $e^{\prime} q$ to a subprojection of $e^{\prime} p$.

PROOF. There is a central projection $e$ such that $p-p \cap q \lesssim q-p \cap q$ on $e$ and $q-p \cap q \lesssim p-p \cap q$ on $e^{\prime}$. If $p-p \cap q \sim r \leqq q-p \cap q$ on $e$ then $p-p \cap q$ and $r$ are perspective in their union by Lemma 3. Since this union is orthogonal to $p \cap q$, Lemma 2 implies that $p$ and $p \cap q+r$ are perspective on $e$. Similarly on $e^{\prime}$ we have $q$ subperspective to $p$.

The relation of perspectivity is, in general, not even finitely additive. However, the following improvement of Lemma 2 is valid in 
any orthomodular lattice. If $a_{i}$ and $b_{i}$ are perspective in $\left[0, e_{i}\right]$ for $i \in I$ and the $e_{i}$ are pairwise orthogonal, then $\sum a_{i}$ and $\sum b_{i}$ are perspective. $^{2}$ Again, it follows directly from Lemma 2 that if the projections $p=\sum p_{i}$ and $q=\sum q_{i}$ are orthogonal and $p_{i}$ and $q_{i}$ are perspective for all $i$, then $p$ and $q$ are perspective.

With regard to possible generalizations, we note that, except for Lemma 4, our arguments are nonspatial, and so apply to $A W^{*}$. algebras. More generally, very little seems to be known concerning perspectivity in orthomodular lattices. M. F. Janowitz has called our attention to an example [1, p. 21] of an orthomodular lattice in which perspectivity fails to be transitive.

\section{BIBLIOGRAPHY}

1. R. P. Dilworth, On complemented lattices, Tôhoku Math. J. 47 (1940), 18-23.

2. Jacob Feldman, Isomorphisms of rings of operators, Thesis, University of Chicago, Chicago, Ill., 1954.

3. Irving Kaplansky, Projections in Banach algebras, Ann. of Math. (2) 53 (1951), 235-249.

4. L. H. Loomis, The lattice theoretic background of the dimension theory of operator algebras, Mem. Amer. Math. Soc. No. 18 (1955), 36 pp.

5. J. von Neumann, Continuous geometry, Princeton Mathematical Series No. 25, Princeton Univ. Press, Princeton, N. J., 1960.

6. D. M. Topping, Jordon algebras of self-adjoint operators, Bull. Amer. Math. Soc. 71 (1965), 160-164.

\section{University of Chicago}

2 This result and Lemma 1 have been obtained independently by S. S. Holland, Jr. in Distributivity and perspectivity in orthomodular lattice, Trans. Amer. Math. Soc. 112 (1964), 330-343. 